\title{
Isolated ureteric metastasis from a primary cervical malignancy
}

\author{
Thumuluru Kavitha Madhuri • Anil Tailor • \\ Simon Butler-Manuel
}

Received: 11 December 2009 / Accepted: 15 January 2010/Published online: 23 February 2010

(C) Springer-Verlag 2010

\begin{abstract}
Cervical cancer globally accounts for more than 250,000 deaths per year. Metastasis occurs through local extension and lymphatic dissemination. Ureteric metastasis is extremely rare and there are $<10$ cases reported in the English literature. We present an interesting case of ureteric metastasis arising from a primary cervical malignancy 5 years after initial treatment which highlights the role of careful history taking and examination in patients with previous malignancy.
\end{abstract}

Keywords Primary cervical malignancy .

Ureteric metastasis · Laparoscopy

\section{Introduction}

Globally, cervical cancer (CC) accounts for more than 250,000 deaths annually. Traditionally, early stage disease is treated by surgery but chemoradiotherapy is the treatment of choice for disease not limited to the cervix. Metastasis is widely known to frequently occur through local extension and lymphatic spread (dissemination). Ureteric metastasis from cervical malignancy is extremely rare with $<10$ cases reported in the English literature. We report an interesting case of ureteric metastasis arising from a primary cervical malignancy 5 years following initial treatment.

T. K. Madhuri $(\bowtie) \cdot$ A. Tailor $\cdot$ S. Butler-Manuel Department of Gynaecological Oncology,

The Royal Surrey County Hospital,

Level B, GOPD, Egerton Road,

Guildford, Surrey GU2 7XX, UK

e-mail: docmadhuri231@doctors.org.uk

\section{Case summary}

A 44-year-old multiparous lady was referred from her local hospital 5 years following her original referral with a severely dyskaryotic smear. She was diagnosed with a grade 3 International Federation of Gynecology and Obstetrics (FIGO) stage 1b squamous cell carcinoma of the cervix with lymphoepithelioma-like growth pattern and had been treated by a radical abdominal hysterectomy and bilateral pelvic lymph node dissection (PLND) with conservation of ovaries at the patient's request. Histology confirmed the tumor was confined to the cervix measuring $9 \mathrm{~mm}$ in horizontal dimension and more than $5 \mathrm{~mm}$ in depth with lymphovascular space invasion present. All the 29 pelvic lymph nodes harvested (11 from the left and 18 from the right) were clear of disease and no residual disease was present in the hysterectomy specimen. No adjuvant treatment was given and she was routinely followed up for 5 years.

She then complained of right iliac fossa pain and was investigated with imaging. Ultrasound revealed a $3-\mathrm{cm}$ mass near the right kidney which was causing hydronephrosis. She underwent percutaneous stenting of her right ureter. Computed tomography (CT) scan showed a right-sided 6- to 7-cm cystic area at the level of the pelvic brim obvious of disease recurrence with no evidence of lymphadenopathy. Following a multidisciplinary team (MDT) meeting, she was offered combination chemoradiotherapy. Chemotherapy consisted of cisplatin and 5-fluorouracil followed by external beam radiotherapy to the pelvis including the lower paraaortic region. Repeat imaging following completion of treatment showed persistence of a small cystic area lateral to the ureteric stent (Fig. 1). The rest of the pelvis was clear of disease. As she had a very good response to treatment, MDT recommended resection of residual mass in the right pelvis. This was in keeping with the patient's wishes and 


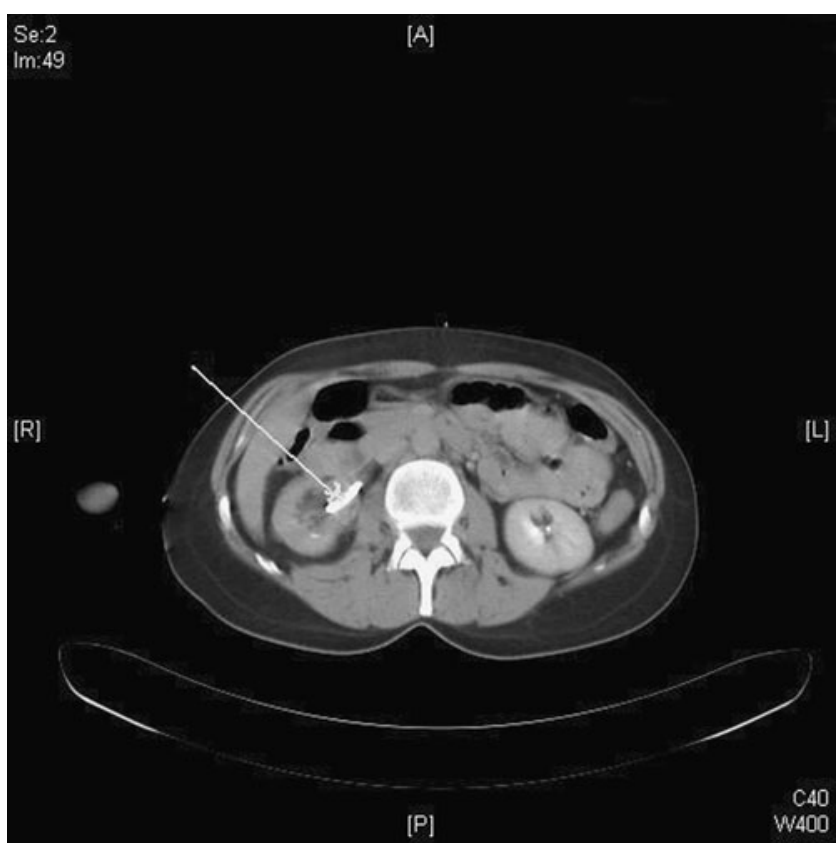

Fig. 1 CT image following completion of treatment with arrow highlighting persistence of a small cystic area lateral to the ureteric stent

she elected to have a bilateral salpingo-oophorectomy (BSO) with biopsies of suspicious areas. She underwent a laparotomy which included a BSO, pelvic and para-aortic node dissection, and resection and re-anastomosis of $20 \mathrm{~mm}$ of her right pelvic ureter which was involved in the mass. She was discharged following a routine postoperative recovery. Histology showed benign bilateral ovarian cysts, with no evidence of metastatic disease in all the nodes removed with evidence of extensive fibrosis. The proximal one third of the excised segment of the ureter showed poorly differentiated adenocarcinoma contained within the ureteric wall with no extension to the mucosa or the circumferential margin and changes consistent with previous chemoradiotherapy. The carcinoma consisted of a small foci contained entirely within the ureteric wall without any extension to the mucosa or circumferential margin with clear surgical resection margins.

In view of clear resection margins and recent extensive treatment, expectant policy was adopted. She presented repeatedly with urinary tract infections and the right ureteric stent was removed following cystoscopy. Ten months later, at routine follow-up, an inflamed looking skin lesion $1.5 \mathrm{~cm}$ in diameter was noticed on her right flank adjacent to the skin scar from the ureteric stent, highly suspicious of an intradermal metastasis. Excision biopsy was performed and histology reported as adenosquamous differentiation in a squamous cell carcinoma possibly following therapy. She remained well for 6 months but complained again of right iliac fossa pain. CT scan showed metastatic disease in the liver and right kidney. She declined any further treatment and died of progressive disease 9 months later.

\section{Discussion}

$\mathrm{CC}$ is the second most common gynecological malignancy and a leading cause of death in young women in the developing world [1]. Globally, it accounts for more than 250,000 deaths annually with more than two thirds of these in women in developing countries presenting with late stage disease $[2,3]$. This disparity is explained by the success of the cervical screening program in most developed countries leading to identification of precancerous or early cervical malignancies [4].

Treatment of CC is based on the stage and grade of disease. FIGO staging is usually clinical based on anatomical disease extent and involvement. Management of early stage CC (FIGO stages IA and IB) is usually surgical. Standard treatment for IA2 and IB1 is by radical hysterectomy + bilateral PLND. The outcome following surgery is associated with a variety of prognostic factors including tumor volume, depth of stromal invasion, presence or absence of lymph-vascular space invasion (LVSI) and proximity to vaginal and parametrial margins. A subset of patients will require chemoradiotherapy following surgery (trimodality treatment) for risk factors such as positive lymph nodes, close $(<3 \mathrm{~mm})$ or positive surgical resection margins, and deep stromal invasion with possible LVSI. Chemoradiotherapy is the treatment of choice for stage $2 b$ disease or greater [5].

Recurrence rates in CC have been reported as $10-20 \%$ for stages Ib to IIa and as high as $50-70 \%$ in locally advanced cases (IIb-IVa) [6]. Recurrent disease is associated with poor prognosis and a 10-20\% 1-year survival and chemotherapy is merely palliative [6]. Metastasis from CC is primarily through local extension or lymphatic spread. Hematogenous spread though rare is the commonest cause of distant metastasis commonly to the liver, lung, and bone [7].

Malignant ureteric involvement can be by primary or secondary disease. Solitary ureteric metastasis from CC is rare. In this case, it heralded the onset of disseminated disease after a long disease-free interval. The first documented metastasis to the ureter was by Stow in 1909 [8]. The ureter is usually involved by direct extension commonly to the lower part of the ureter unlike our case where the proximal on third was involved [9]. Encasement, invasion, and displacement are the common manifestations of secondary involvement and will inevitably interfere with ureteric function and have an obstructive effect on the renal tract as in our case demonstrated by hydronephrosis [10].

Early clinical stage at initial diagnosis did not protect against the development of subsequent metastasis. The 
patient was clinically free of disease for nearly 5 years after treatment. Symptoms in patients with previous cervical malignancy should not be ignored.

Conflict of interest There is no actual or potential conflict of interest in relation to this article.

\section{References}

1. Jemal A et al (2008) Cancer statistics 2008. CA Cancer J Clin 58 (2):71-96

2. Sankaranarayanan R, Ferlay J (2006) Worldwide burden of gynaecological cancer: the size of the problem. Best Pract Res Clin Obstet Gynaecol 20(2):207-225
3. Castellsagué X (2008) Natural history and epidemiology of HPV infection and cervical cancer. Gynecol Oncol 110:S4-S7

4. Kehoe S (2006) Treatments for gynaecological cancers. Best Pract Res Clin Obstet Gynaecol 20(6):985-1000

5. Kesic V (2006) Management of cervical cancer. Eur J Surg Oncol 32(8):832-837

6. Pectasides D, Kamposioras K, Papaxoinis G, Pectasides E (2008) Chemotherapy for recurrent cervical cancer. Cancer Treat Rev 34:603-613

7. Carlson V, Delclos L, Fletcher GH (1967) Distant metastases in squamous-cell carcinoma of the uterine cervix. Radiology 88:961-966

8. Stow B (1909) Fibrolymphosarcomata of both ureters, metastatic to a primary lymphosarcoma of the anterior mediastinum of thymus origin. Ann Surg 50:901

9. Richie JP, Withers G, Ehrlich RM (1979) Ureteral obstruction secondary to metastatic tumors. Surg Gynecol Obstet 148(3):355-357

10. Van Blerk PJ (1983) Malignant involvement of the ureter. Surg Annu 15:261-282 